occasions; in none of these cases was $S$ dysenteriae type 1 the causative organism. Two patients had bacteraemia. ${ }^{1-3}$ Petechial rashes have been seen on some occasions in shigellosis, ${ }^{4}$ but they are quite different from rose spots-which have sharply defined borders and disappear on applying pressure. The size of the spots and absence of itching differentiate them from allergic rashes due to ampicillin. An upsurge of shigellosis due to $S$ dysenteriae 1 has been recorded in Bangladesh since the end of war in $1971 .^{5}$ Nevertheless, rose spots were not detected in any one of over 4000 patients seen during the last five years.

Correspondence should be addressed to: $\operatorname{Dr} M$ Mujibur Rahaman, Cholera Research Laboratory, GPO Box 128, Dacca 2, Bangladesh.

${ }^{1}$ Ghon, A, and Roman, B, Wiener klinische Wochenschrift, 1915, 2, 579.

2 Brunaer, S, Wiener klinische Wochenschrift, 1916, 5, 128.

3 Barrett, O, O'Connor, E, and Connor, J, American fournal of Tropical Medicine, 1969, 18, 555.

${ }^{4}$ Goscienski, P J, and Haltalin, K C, American fournal of Diseases of Children, $1970,119,152$. 1975, 131, 700 .

(Accepted 30 May 1977)

Cholera Research Laboratory, Mohakhali, Dacca 12, Bangladesh

$M$ MUJIBUR RAHAMAN, MB, PHD, head of clinical division

A K M JAMIUL ALAM, MBBS, chief physician of clinical division
${ }^{5}$ Rahaman, M M, Huq, I, and Dey, C R, The fournal of Infectious Diseases,

A retrospective analysis of 200 patients showed 20 instances of fanshaped shadows in the area where the catheter tip had been located. In two patients we found two or three shadows associated with different positions of the catheter tip. The clinical course had always been uncomplicated, and the radiological signs had regressed rapidly.

To prevent thromboembolisms, we administered heparin $(50 \mathrm{mg}$ every 6 hours) whenever a Swan-Ganz catheter was used. In addition, $\mathrm{KCl}$ was no longer given through the pulmonary artery lumen. In a subsequent series of 201 patients, we found only nine fan-shaped shadows. Heparin had thus halved the incidence of fan-shaped shadows, but they had not been eradicated, suggesting mechanisms other than thromboembolism for their genesis.

Mechanical irritation of the blood vessel by the to-and-fro movements of the catheter tip, or tear of the wall of an arteriole by an excessively inflated balloon ${ }^{4}$ could also cause some fan-shaped shadows. This would explain why most (24/29) of the observed shadows were located close to the hilum.

Heparin treatment seems advisable whenever pulmonary artery monitoring is carried out: we have not observed a single haemorrhagic complication in our patient population, and the incidence of fanshaped shadows was decreased by half

Address for correspondence: F Hagemeijer, Thoraxcenter, Erasmus University, PB 1738, Rotterdam, The Netherlands.

1 Foote, G A, Schabel, S I, and Hodges, M, New England fournal of Medicine, 1974, 290, 927 .

${ }^{2}$ Goodman, D J, et al, New England fournal of Medicine, 1974, 291, 777.

3 Kateman, I, et al, 15e Federatie Vergadering Nijmegen (The Netherlands), p 224, 1974

4 Chun, G M H, and Ellestad, M H, New England fournal of Medicine, 1972, 284, 1041.

5 Golden, M S, et al, American fournal of Cardiology, 1973, 32, 865.

(Accepted 1 fune 1977)

\section{Fan-shaped shadows due to pulmonary artery catheters: heparin prophylaxis}

Since 1971 we have monitored all our patients with myocardial infarction with a Swan-Ganz catheter $(5 \mathrm{~F}$ single lumen or $7 \mathrm{~F}$ thermodilution catheter) positioned in the pulmonary artery. If an AP chest roentgenogram showed that the catheter tip was located too far in the lung fields, the catheter was pulled back $5-10 \mathrm{~cm}$. The lumen was used for intermittent pressure measurements, blood sampling, and intravenous drug administration; it was continuously flushed with glucose $5 \%$ in water (with lidocaine added when needed).

By early 1974 we had become aware of curious fan-shaped shadows occasionally observed in the lung fields (fig). ${ }^{1-3}$ These sometimes resembled the triangular wedge found in pulmonary embolism, but we observed no other symptoms of this condition.

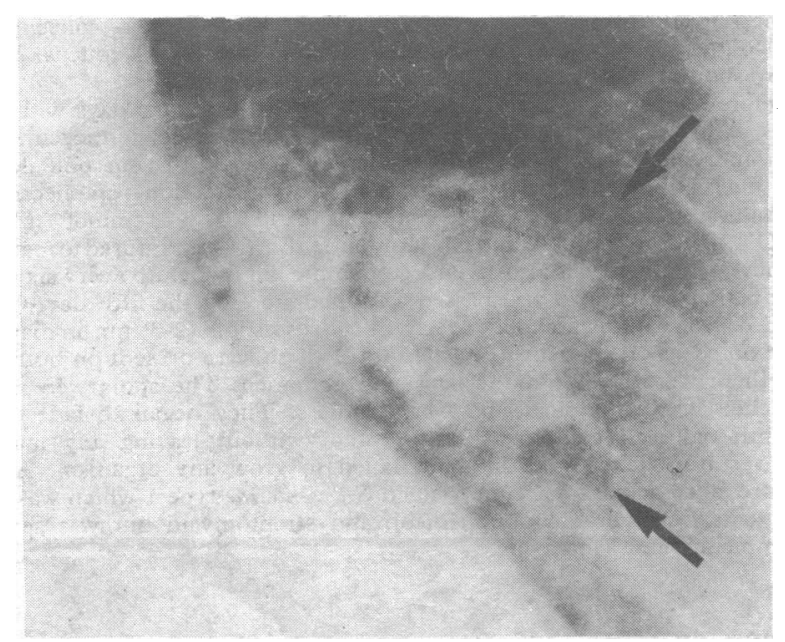

Left parahilar fan-shaped shadow (arrow) at the location of the tip of a Swan-Ganz catheter two days previously. This shadow disappeared over 10 days.
Thoraxcenter, Erasmus University, Rotterdam, The Netherlands FRANS HAGEMEIJER, MD, consultant cardiologist COK J STORM, MD, senior registrar

\section{Multiple small bowel strictures in a child and accidental potassium chloride ingestion}

In the past decade it has become well recognised that enteric-coated potassium chloride tablets may cause strictures of the small bowel. ${ }^{1}$ Experimental and clinical studies have shown that these strictures result from the rapid release of potassium chloride when the enteric coat disintegrates in the alkaline medium of the small intestine. ${ }^{2}$ Despite the fact that slow release, non-enteric coated preparations of potassium chloride are virtually free of this complication, ${ }^{3}$ entericcoated preparations are still marketed and new cases of intestinal stricture continue to be reported, ${ }^{4}$ typically in adults on long-term medication. We have failed to find any report of this complication in a child.

\section{Case report}

A 2-year-old boy was admitted to this hospital about 16 hours after swallowing an unknown quantity of his grandmother's "heart tablets." Gastric lavage retrieved four tablets, which were identified as Salupres, a combination of reserpine and hydrochlorothiazide around an enteric core of potassium chloride. He was discharged home after two uneventful days of observation, but readmitted ten days later with a 24-hour history of abdominal colic and bile-stained vomiting. An abdominal $x$-ray film showed a complete low small bowel obstruction. At laparotomy this obstruction was found to be caused by omental bands, which were adherent at two points to the antimesenteric border of the terminal ileum. Division of the bands relieved the obstruction, but at both these points the ileum showed a stricture to half its normal diameter for a length of some $3 \mathrm{~mm}$. Two further similar strictures were found more proximally in the ileum, one of which lay at the apex of a short, non-obstructive, intussusception, which was easily reduced. It was elected not to resect the strictures since, firstly, they were not in themselves obstructive, and secondly the natural history of these early strictures is unknown. 